\title{
SIR Balancing for Strongly Connected Interference Networks - Existence and Uniqueness of a Solution
}

\author{
Martin Schubert *, Nikola Vucic *, Holger Boche *\# \\ \# Fraunhofer Institute for Telecommunication, \\ Heinrich-Hertz Institute (HHI), \\ Einsteinufer 37, \\ 10587 Berlin, Germany \\ * Fraunhofer German-Sino Lab for Mobile Communications (MCI) \\ Einsteinufer 37, \\ 10587 Berlin, Germany
}

\begin{abstract}
In this paper we revisit the problem of SIR Balancing which was extensively studied in the wireless communication literature. We generalize existing results by showing conditions for the existence and uniqueness of a max-min optimizer. It is shown that a unique positive optimizer exists if the underlying asymptotic coupling matrix is irreducible and the interference functions are strictly monotonic on the dependency set. We also show how the solution can be extended to include power constraints.
\end{abstract}

\section{INTRODUCTION}

One trend in wireless communications is towards high user density. However, interference puts a limit on how many users per area can be served at a certain data rate. Assigning each user a separate resource is not always an efficient way of organizing the system. If the number of users is high then each user only gets a small fraction of the overall resource. Shortages occur, especially when many users have high data rate requirements. So the classical design paradigm of independent point-to-point communication links is gradually being replaced by a network-centric point of view. In order to exploit the network to its full potential, interference should be tolerated, but in a controlled way. Dynamic interference management and resource allocation are expected to play a key role in this respect.

However, interference between users complicates the analysis and optimization of the network. The performance of any user can depend on the transmission strategies of other users. This typically leads to joint problem formulations with performance measures that account for all users.

Previous Work. One fundamental problem in this context is to balance the signal-to-interference ratios (SIR). First results on this problem already appeared decades ago (e.g. [1]). In this early work, the interference between users was modeled by a

The authors are supported in part by the Bundesministerium für Bildung und Forschung $(B M B F)$ under grant 01SF0708 (project TEROPP). Parts of this work have been performed in the framework of the European research project SAPHYRE, which is partly funded by the European Union under its FP7 ICT Objective 1.1 - The Network of the Future. linear function of the transmission powers. It was observed that irreducibility of the coupling matrix is the essential property that enables many results. Existence and uniqueness is a direct consequence of the Perron-Frobenius theorem. Linear interference functions are a standard model in power control theory.

The analysis becomes more complicated for systems with non-linear interference functions. An example is joint power control and multiuser beamforming, where the coupling matrix depends on the beamformers, which in turn depend on the powers (details are given in the appendix). In this context, the max-min SIR balancing problem was studied in [2], [3]. Also in this case, the irreducibility of the coupling matrix is an important requirement.

Another line of research is studying interference coupling from a more general and abstract perspective, based on $a x$ iomatic interference functions [4]. In this work, interference is modeled as monotonic and scale-invariant (homogeneous) functions. It turns out that general interference functions do not have sufficient structure to ensure the existence of a max-min optimal power vector. It is thus important to study additional properties. For example, it was shown in [5] that the problem becomes tractable under the additional assumption of concavity.

Contributions. In this paper we study the SIR balancing problem within the general framework of monotonic and scale-invariant interference functions, without the assumption of convexity or concavity. In order to ensure existence and uniqueness of an optimizer, we require that the system is fully connected in terms of its asymptotic coupling matrix [6]. These properties are sufficient to ensure the existence of a positive optimizer. Then, uniqueness is shown under the additional assumption of strict monotonicity. Finally, we show how the framework can be extended by noise and power constraints.

Notation. Matrices and vectors are denoted by upper-case and lower-case boldface letters, respectively. Let $\boldsymbol{y}$ be a vector, then $y_{l}=[\boldsymbol{y}]_{l}$ is the $l$ th component. Likewise, $A_{m n}=[\boldsymbol{A}]_{m n}$ 


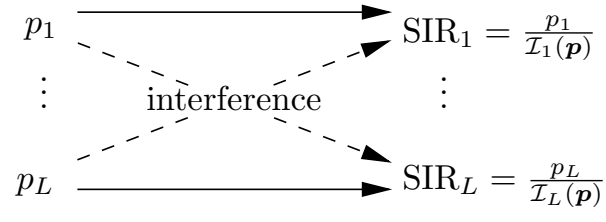

Fig. 1. Interference-coupled system.

is a component of the matrix $\boldsymbol{A}$. A vector inequality $\boldsymbol{x}>\boldsymbol{y}$ means $x_{k}>y_{k}$, for all $k$, and $\boldsymbol{y}>0$ means componentwise greater zero. Inequality $\boldsymbol{y} \geq \boldsymbol{x}$ means $y_{l} \geq x_{l}$ for all components. The notation $\boldsymbol{y} \supsetneqq \boldsymbol{x}$ means $\boldsymbol{y} \geq \boldsymbol{x}$ and there is at least one component $l$ such that $y_{l}>x_{l}$. Finally, $\boldsymbol{y} \neq \boldsymbol{x}$ means that inequality holds for at least one component. The sets of non-negative reals and positive reals are denoted by $\mathbb{R}_{+}$and $\mathbb{R}_{++}$, respectively.

\section{A. Axiomatic SIR Balancing}

Consider a system with $L$ communication links with respective transmission powers $\boldsymbol{p}=\left[p_{1}, p_{2}, \ldots, p_{L}\right]^{T} \in \mathbb{R}_{+}^{L}$. The system is illustrated in Fig. 1. Following the related literature, we will also refer to the links as "users". However, it should be noted that $p_{l}$ can also model other system variables. An example will be given in Section III.

The impact of $\boldsymbol{p}$ on some link $l$ is characterized by a socalled interference function $\mathcal{I}_{l}(\boldsymbol{p})$. A very simple and basic interference model is the following framework of axioms [4]

Definition 1. We say that $\mathcal{I}: \mathbb{R}_{+}^{L} \mapsto \mathbb{R}_{+}$is an interference function if the following axioms are fulfilled:
A1 (positivity)
There is a $\boldsymbol{p}>0$ with $\mathcal{I}(\boldsymbol{p})>0$
A2 (scale invariance) $\mathcal{I}(\alpha \boldsymbol{p})=\alpha \mathcal{I}(\boldsymbol{p})$ for all $\alpha>0$
$\mathbf{A 3}$ (monotonicity) $\quad \mathcal{I}(\boldsymbol{p}) \geq \mathcal{I}\left(\boldsymbol{p}^{\prime}\right) \quad$ if $\boldsymbol{p} \geq \boldsymbol{p}^{\prime}$

An example is given in the appendix.

A fundamental performance measure is the signal-tointerference ratio

$$
\operatorname{SIR}_{l}(\boldsymbol{p})=\frac{p_{l}}{\mathcal{I}_{l}(\boldsymbol{p})} .
$$

The problem under consideration is the so-called max-minSIR-balancing problem

$$
\sup _{\boldsymbol{p} \in \mathbb{R}_{++}^{L}} \min _{1 \leq l \leq L} \frac{\operatorname{SIR}_{l}(\boldsymbol{p})}{\gamma_{l}} .
$$

The power vector $\boldsymbol{p}$ is assumed to be contained in $\mathbb{R}_{++}^{L}$. This is justified by our design goal, which is to balance the SIR values. A solution of (2) cannot contain a zero power. All users contribute, there are no inactive users because a solution containing $p_{k}=0$ would mean that all SIR are zero. The values $\gamma \in \mathbb{R}_{++}^{L}$ can be regarded as weighting factors which are chosen according to individual priorities.

\section{B. Strongly Connected System}

Some basic properties of problem (2) were already studied in [4]. Here, we extend these results by assuming that the links are "strongly connected". This is defined by the following properties.

In order to model whether an interference function depends on some resource or not, we introduce the coupling matrix $\boldsymbol{A}_{\mathcal{I}}$, which characterizes the interference coupling between the users.

The asymptotic coupling matrix $\boldsymbol{A}_{\mathcal{I}}$ is defined as follows. Let $\boldsymbol{e}_{l}$ be the all-zero vector with the $l$-th component set to one. Then,

$$
\left[\boldsymbol{A}_{\mathcal{I}}\right]_{k l}= \begin{cases}1 & \text { if there exists a } \boldsymbol{p}>0 \text { such that } \\ & \lim _{\delta \rightarrow \infty} \mathcal{I}_{k}\left(\boldsymbol{p}+\delta \boldsymbol{e}_{l}\right)=+\infty \\ 0 & \text { otherwise }\end{cases}
$$

If there is one $\boldsymbol{p}$ that fulfills the condition in (3), then this condition is fulfilled for all $\boldsymbol{p}>0$.

Throughout this paper, we assume that $\boldsymbol{A}_{\mathcal{I}}$ is irreducible. A non-negative $L \times L$ matrix $\boldsymbol{A}_{\mathcal{I}}$ is said to be irreducible if and only if its directed graph $\mathcal{G}\left(\boldsymbol{A}_{\mathcal{I}}\right)$ is strongly connected. This is illustrated by the following example.

$$
\boldsymbol{A}_{\mathcal{I}}=\left[\begin{array}{llll}
0 & 0 & 0 & 1 \\
1 & 0 & 0 & 0 \\
1 & 1 & 0 & 0 \\
1 & 1 & 1 & 0
\end{array}\right] \quad \mathcal{G}\left(\boldsymbol{A}_{\mathcal{I}}\right):
$$

The graph $\mathcal{G}\left(\boldsymbol{A}_{\mathcal{I}}\right)$ consists of $L=4$ nodes $N_{1}, \ldots, N_{L}$. A pair of nodes $\left(N_{i}, N_{j}\right)$ is connected by a directed edge if $\left[\boldsymbol{A}_{\mathcal{I}}\right]_{i j}>$ 0. A graph is called strongly connected if for each pair of nodes $\left(N_{i}, N_{j}\right)$ there is a sequence of directed edges leading from $N_{i}$ to $N_{j}$.

The dependency set of link $k$ is

$$
\mathrm{L}_{k}=\left\{l \in[1,2, \ldots, K]:\left[\boldsymbol{A}_{\mathcal{I}}\right]_{k l}=1\right\} .
$$

The set $L_{k}$ is non-empty. Because of the assumed irreducibility there is at least one non-zero entry in each row and column.

$\mathcal{I}_{k}(\boldsymbol{p})$ is said to be strictly positive if for any $\boldsymbol{p} \geq 0$ with $p_{l}>0$ for some $l \in \mathrm{L}_{k}$ we have $\mathcal{I}_{k}(\boldsymbol{p})>0$.

Definition 2. A system consisting of $L$ interference functions is said to be strongly connected if the functions are strictly positive and $\boldsymbol{A}_{\mathcal{I}}$ is irreducible.

\section{UNCONSTRAINED SIR BALANCING}

In this section we consider a strongly connected system with powers $\boldsymbol{p} \in \mathbb{R}_{++}^{L}$. Power constraints will be discussed later, in Section III.

Since all involved quantities are positive, we have

$$
\begin{aligned}
\sup _{\boldsymbol{p} \in \mathbb{R}_{++}^{L}} \min _{1 \leq k \leq L} \frac{p_{k}}{\gamma_{k} \mathcal{I}_{k}(\boldsymbol{p})}=\frac{1}{\inf _{\boldsymbol{p} \in \mathbb{R}_{++}^{L}}\left(\min _{1 \leq k \leq L} \frac{p_{k}}{\gamma_{k} \mathcal{I}_{k}(\boldsymbol{p})}\right)^{-1}} \\
=\frac{1}{\inf _{\boldsymbol{p} \in \mathbb{R}_{++}^{L}}\left(\max _{1 \leq k \leq L} \frac{\gamma_{k} \mathcal{I}_{k}(\boldsymbol{p})}{p_{k}}\right)}
\end{aligned}
$$

Thus, the optimum of the SIR balancing problem is $1 / C(\gamma)$ and both problems are equivalent in terms of optimizers (if 
existent). Thus, instead of studying the max-min SIR balancing problem directly, we analyze the following problem instead.

$$
C(\boldsymbol{\gamma})=\inf _{\boldsymbol{p} \in \mathbb{R}_{++}^{L}} \max _{1 \leq k \leq L} \frac{\gamma_{k} \mathcal{I}_{k}(\boldsymbol{p})}{p_{k}} .
$$

All results can be transferred directly to the original problem.

It was shown in [4, Thm. 2.7] that there always exists a $\boldsymbol{p}^{*} \supsetneqq 0$, such that

$$
p_{k}^{*} \cdot C(\gamma)=\gamma_{k} \mathcal{I}_{k}\left(\boldsymbol{p}^{*}\right), \quad 1 \leq k \leq K .
$$

This result holds for all interference functions fulfilling A1A3.

\section{A. The Strongly Connected Case}

Under the assumption of a strongly connected system, we show the following result.

Theorem 1. If the system is strongly connected then we have $C(\gamma)>0$ and any $\boldsymbol{p}^{*} \geq 0$ fulfilling (6) is strictly positive, i.e., $\boldsymbol{p}^{*}>0$.

Proof: First, we show $C(\gamma)>0$. Suppose that $C(\gamma)=0$, then with (6) all interference functions must be zero. However, the assumed irreducibility yields a contradiction. The solution $\boldsymbol{p}^{*} \supsetneqq 0$ has at least one non-zero entry. Because of irreducibility, we know that there is at least one link $k$ with $\mathcal{I}_{k}\left(\boldsymbol{p}^{*}\right)>0$.

Next, we show $\boldsymbol{p}^{*}>0$. We know from (6) that there is an index $k_{0}$ such that $r_{k_{0}}^{*}>0$. This affects all other links in $\mathrm{L}_{k_{0}}$. That is, $\mathcal{I}_{l}\left(\boldsymbol{p}^{*}\right)>0$ for all $l \in \mathrm{L}_{k_{0}}$. From (6) it follows that $p_{l}^{*}>0$ for all $l \in \mathrm{L}_{k_{0}}$. These powers affect in turn other links in their dependency set. Because of irreducibility, we know that all components of the vector $p^{*} \geq 0$ fulfilling (6) must be strictly positive.

Corollary 1. If the system is strongly connected then the maxmin problem (2) has a solution $\boldsymbol{p}^{*}>0$.

Proof: From Theorem 1 we know that there always is a $\boldsymbol{p}^{*}>0$ that balances the SIR at a level $C(\gamma)>0$. That is, $\boldsymbol{p}^{*}$ attains the infimum $C(\gamma)=\max _{k} \gamma_{k} \mathcal{I}_{k}\left(\boldsymbol{p}^{*}\right) / p_{k}^{*}$. Also, we know from [4, Thm. 2.14] that $C(\gamma)$ is the only possible balanced level.

Thus far, we have shown the existence of a solution $\boldsymbol{p}^{*}>0$ but not uniqueness. There possibly are several solutions. Uniqueness will be shown in the next section under the additional assumption of strict monotonicity.

\section{B. Strict Monotonicity implies a Unique Optimizer}

We begin with a definition.

Definition 3 (strict monotonicity). $\mathcal{I}_{k}(\boldsymbol{p})$ is said to be strictly monotonic if for arbitrary $\boldsymbol{p}^{(1)}, \boldsymbol{p}^{(2)}$, the inequality $\boldsymbol{p}^{(1)} \geq$ $\boldsymbol{p}^{(2)}$, with $p_{l}^{(1)}>p_{l}^{(2)}$ for some $l \in \mathrm{L}_{k}$, implies $\mathcal{I}_{k}\left(\boldsymbol{p}^{(1)}\right)>$ $\mathcal{I}_{k}\left(\boldsymbol{p}^{(2)}\right)$.

Theorem 2. If the interference functions are strongly connected and strictly monotonic, then the set of equations (6) has a unique solution $\boldsymbol{p}^{*}>0$, up to scalar multiples.
Proof: The proof is by contradiction. Assume that there is another vector $\hat{\boldsymbol{p}}>0$ that also fulfills (6), and $\hat{\boldsymbol{p}}$ is not a scalar multiple of $\boldsymbol{p}^{*}$. Equation (6) is invariant with respect to a scaling of its solutions, thus we can assume $\hat{\boldsymbol{p}} \supsetneqq \boldsymbol{p}^{*}$ without loss of generality. By $J^{(=)}$we denote the set of indices for which the inequality is fulfilled with equality. Likewise, $J^{(>)}$denotes the set of indices for which the inequality is strict. Because of the irreducibility of $\boldsymbol{A}_{\mathcal{I}}$ we can always find a $k \in J^{(=)}$and a $l \in J^{(>)}$such that $l \in \mathrm{L}_{k}$. We have

$$
\begin{aligned}
& \hat{p}_{k}=p_{k}^{*}, \\
& \hat{p}_{l}>p_{l}^{*} \quad l \in \mathrm{L}_{k} .
\end{aligned}
$$

Because of strict monotonicity we have $\mathcal{I}_{k}(\hat{\boldsymbol{p}})>\mathcal{I}_{k}\left(\boldsymbol{p}^{*}\right)$. With (6) we obtain the contradiction

$$
\gamma_{k} \mathcal{I}_{k}\left(\boldsymbol{p}^{*}\right)=p_{k}^{*} \cdot C(\gamma)=\hat{p}_{k} \cdot C(\gamma)=\gamma_{k} \mathcal{I}_{k}(\hat{\boldsymbol{p}}) .
$$

The next result connects equation (6) and the balancing problem (5).

Theorem 3. If the interference functions are strongly connected and strictly monotonic, then the SIR balancing problem (5) has an optimizer $\boldsymbol{p}^{*}>0$, unique up to a scalar multiple, that balances all the ratios $\mathrm{SIR}_{k} / \gamma_{k}$ at the level $C(\gamma)$, i.e.,

$$
\begin{aligned}
C(\boldsymbol{\gamma}) & =\min _{\boldsymbol{p} \in \mathcal{R}_{++}^{L}}\left(\max _{1 \leq l \leq L} \frac{\gamma_{l} \mathcal{I}_{l}(\boldsymbol{p})}{p_{l}}\right) \\
& =\frac{\gamma_{1} \mathcal{I}_{1}\left(\boldsymbol{p}^{*}\right)}{p_{1}^{*}}=\cdots=\frac{\gamma_{L} \mathcal{I}_{L}\left(\boldsymbol{p}^{*}\right)}{p_{L}^{*}}
\end{aligned}
$$

Proof: From Corollary 1 we know that an optimizer exists. Now we show that any optimizer balances the SIR values at the level $C(\gamma)$. The proof is by contradiction. Let $\boldsymbol{p}^{*}>0$ be an arbitrary optimizer, and assume that there is an index $k_{1}$ such that $\gamma_{k_{1}} \mathcal{I}_{k_{1}}\left(\boldsymbol{p}^{*}\right) / p_{k_{1}}^{*}<C(\boldsymbol{\gamma})$. We can decrease $p_{k_{1}}^{*}$, while preserving strict inequality. Because of irreducibility and strict monotonicity, any decrease of $p_{k_{1}}^{*}$ reduces the interference of some other user $k_{2}$. Thus, we obtain a new vector $\boldsymbol{p}^{\prime} \leq \boldsymbol{p}^{*}$ with $\gamma_{k_{2}} \mathcal{I}_{k_{2}}\left(\boldsymbol{p}^{\prime}\right) / p_{k_{2}}^{\prime}<C(\gamma)$. Now, users $k_{1}$ and $k_{2}$ are below $C(\gamma)$. We go on decreasing $p_{k_{2}}^{\prime}$, while preserving strict inequality. This reduces the interference at other users, which in turn can reduce their powers. By the assumption of irreducibility, all users are included after a finite number of steps. That is, all other users can benefit from the reduction of some link power, either directly or indirectly. Thus, it would be possible to achieve a value below the infimum, which is a contradiction, thus proving that an optimizer $\boldsymbol{p}^{*}>0$ fulfills (10). From Theorem 2 we know that this balanced solution is unique up to a scalar multiple.

\section{CONSTRAined POWERS}

In this section we show how the results can be extended to include power constraints, which is important for most practical interference scenarios.

Thus far, we have analyzed the SIR, which is invariant with respect to a scaling, i.e., $\operatorname{SIR}_{l}(\alpha \boldsymbol{p})=\operatorname{SIR}_{l}(\boldsymbol{p})$ for all $\alpha>0$. 
Thus, constraining the norm of $\boldsymbol{p}$ has no effect. This is typical for a system with no noise.

In order to model power-constrained system with noise, the framework of standard interference functions [7] is often used. This framework is also based on a set of axioms similar to A1A3, except that scale invariance (A2) is replaced by scalability $\mathcal{I}(\alpha \boldsymbol{p})<\alpha \mathcal{I}(\boldsymbol{p})$ for all $\alpha>1$. In the literature there are many examples of a successful application of standard interference functions.

In the following we demonstrate that power-constrained SI(N)R balancing can be understood as a special case of problem (2). Our approach is based on the introduction of an auxiliary interference function, which ensures that certain power constraints are fulfilled.

In order for a power constraint to have any effect, it is necessary to introduce noise. In this section we consider $K$ user powers $p_{1}, \ldots, p_{K}$ and a noise power $\sigma_{n}^{2}$. These powers are stacked in an extended power vector

$$
\underline{\boldsymbol{p}}=\left[p_{1}, \ldots, p_{K}, \sigma_{n}^{2}\right]^{T} \in \mathbb{R}_{++}^{K+1} .
$$

In the following we normalize $\sigma_{n}^{2}=1$.

\section{A. Total Power Constraint}

Consider a system with a total power constraint $\sum_{1 \leq k \leq K} \leq$ $P_{\text {max }}$, for some $P_{\text {max }}>0$. In order to enforce this power constraint, we introduce an auxiliary interference function

$$
\mathcal{I}_{K+1}(\underline{\boldsymbol{p}})=\frac{1}{P_{\max }} \cdot \sum_{k=1}^{K} \gamma_{k} \mathcal{I}_{k}(\underline{\boldsymbol{p}}) .
$$

It can be verified that $\mathcal{I}_{K+1}$ fulfills the axioms A1-A3, since the sum of interference functions is an interference function again.

Let $G$ be the dependency matrix of the first $K$ components. We assume that every user causes interference to at least one other user, thus each column of the matrix $\boldsymbol{G}$ has at least one non-zero entry. Also, every $\mathcal{I}_{k}(\boldsymbol{p})$ depends on the noise component. Thus, the $K \times K+1$ asymptotic coupling matrix of the first $K$ interference functions is $[\boldsymbol{G} \mid \mathbf{1}]$, where the last column models the dependency on the noise.

The interference function $\mathcal{I}_{K+1}$ depends on all powers, because of definition (12). Thus, the overall system coupling matrix becomes

$$
\boldsymbol{A}_{\mathcal{I}}=\left[\begin{array}{cc}
\boldsymbol{G} & \mathbf{1} \\
\mathbf{1}^{T} & 1
\end{array}\right]
$$

The matrix $\boldsymbol{A}_{\mathcal{I}}$ is irreducible.

We further assume that the interference functions $\mathcal{I}_{1}, \ldots, \mathcal{I}_{K}$ are strictly monotonic. Since $\sigma_{n}^{2}=1$ is constant, we know that they are also strictly positive. This is a consequence of the properties A1-A3 and strict monotonicity, as observed in [4]. The function $\mathcal{I}_{K+1}$ is the sum of all other interference functions, thus positivity and strict monotonicity holds.

The interference functions $\mathcal{I}_{1}, \ldots, \mathcal{I}_{K}, \mathcal{I}_{K+1}$ constitute a fully connected, strictly monotonic system, and all the results of Section II can be applied. The $K+1$ dimensional SIR balancing problem can be written as

$$
\min _{\underline{\boldsymbol{p}} \in \mathcal{P}}\left(\max _{1 \leq k \leq K+1} \frac{\gamma_{k} \mathcal{I}_{k}(\underline{\boldsymbol{p}})}{p_{k}}\right) .
$$

Here, we optimize over the set

$$
\mathcal{P}=\left\{\underline{\boldsymbol{p}} \in \mathbb{R}_{++}^{K+1}: p_{K+1}=1\right\} .
$$

As a consequence, $\boldsymbol{p}$ always fulfills $p_{K+1}=1$. Recall that the SIR is not affected by a simultaneous scaling of transmission powers and noise, so we could equivalently optimize over the unconstrained set $\mathbb{R}_{++}^{K+1}$. But the problem formulation (14) has the advantage of having a defined noise level.

The additional parameter $\gamma_{K+1}$ can be set to one. Other values will just scale the available power budget.

From Theorem 3 we know that problem (14) has a unique optimizer $\boldsymbol{p}^{*}>0$ such that

$$
\frac{p_{1}^{*}}{\gamma_{1} \mathcal{I}_{1}\left(\underline{\boldsymbol{p}}^{*}\right)}=\cdots=\frac{p_{K}^{*}}{\gamma_{K} \mathcal{I}_{K}\left(\underline{\boldsymbol{p}}^{*}\right)}=\frac{P_{\max }}{\sum_{k=1}^{K} \gamma_{k} \mathcal{I}_{k}\left(\underline{\boldsymbol{p}}^{*}\right)} .
$$

Taking the sum of the first $K$ powers, we obtain the following identity.

$$
\sum_{k=1}^{K} p_{k}^{*}=\sum_{k=1}^{K} \gamma_{k} \mathcal{I}_{k}\left(\underline{\boldsymbol{p}}^{*}\right) \cdot \frac{P_{\max }}{\sum_{k=1}^{K} \gamma_{l} \mathcal{I}_{l}\left(\underline{\boldsymbol{p}}^{*}\right)}=P_{\max }
$$

Hence, all user SIRs are balanced and the sum power constraint is fulfilled with equality.

\section{B. Max-Min SI $(N) R$ Balancing}

Next, consider the functions $Y_{k}(\boldsymbol{p})=\mathcal{I}_{k}(\boldsymbol{p})$ for all $k=$ $1,2, \ldots, K$. For constant noise $p_{K+1}$, the function $Y_{k}$ is a standard interference function [7], characterized by monotonicity and scalability, i.e., $Y(\alpha \boldsymbol{p})<\alpha Y(\boldsymbol{p})$. Moreover, any standard interference function can be written as $\mathcal{I}_{k}(\boldsymbol{p})$ with $p_{K+1}=1$. Thus, both frameworks can be used interchangeably for modeling interference+noise in communication system. For a detailed comparison between the axiomatic framework A1-A3 and the framework of standard interference functions, the reader is referred to [8].

With standard interference functions, the power-constrained SINR balancing problem can be written as follows.

$$
\max _{\boldsymbol{p}>0: \sum_{k=1}^{K} \leq P_{\max }} \min _{k \in \mathcal{K}} \frac{p_{k}}{\gamma_{k} Y_{k}(\boldsymbol{p})} .
$$

Equivalently, we can focus on the problem

$$
C\left(\boldsymbol{\gamma}, P_{\max }\right)=\min _{\boldsymbol{p}>0: \sum_{k=1}^{K} \leq P_{\max }} \max _{k \in \mathcal{K}} \frac{\gamma_{k} Y_{k}(\boldsymbol{p})}{p_{k}} .
$$

Similar as in [9], we can show that

- Problem (19) has a unique optimizer $\boldsymbol{p}^{*}>0$ that balances all SINR at a level $C\left(\gamma, P_{\max }\right)>0$, i.e.,

$$
C\left(\boldsymbol{\gamma}, P_{\max }\right)=\frac{\gamma_{k} Y_{k}\left(\boldsymbol{p}^{*}\right)}{p_{k}^{*}} \quad \text { for all } k=1,2, \ldots, K
$$


- At the optimum, the power constraint is fulfilled with equality, i.e., $\sum_{k=1}^{K} p_{k}^{*}=P_{\max }$.

- $C\left(\gamma, P_{\max }\right)$ is strictly monotonic decreasing in $P_{\max }$.

From the latter property it follows that there cannot be another balanced level. If we find powers $\boldsymbol{p}^{*}>0$ achieving a balanced level, and if this solution fulfills $\sum_{k=1}^{K} p_{k}^{*}=P_{\max }$, then this is the unique optimizer of the SINR balancing problem (19).

Hence, the solution (16) obtained by the SIR balancing approach with an auxiliary interference function yields the unique global optimum of the SINR balancing problem.

\section{CONCLUSiOnS}

We have analyzed the SIR balancing problem and provided conditions for the existence and uniqueness of an optimizer. This analysis provides a common background for existing work in the literature, where the max-min SIR problem was studied under particular assumptions, e.g. concavity, beamforming, linear functions, noise and power constraints, etc. The results of this paper show that any strongly connected system with strictly monotonic interference functions has a unique max-min optimizer.

\section{APPENDIX}

\section{A. Example of an Interference Function}

Consider an uplink system with $K$ single-antenna transmitters and an $M$-element antenna array at the receiver. Independent signals $s_{1}, \ldots, s_{K}$ are transmitted over vectorvalued channels $\boldsymbol{h}_{1}, \ldots, \boldsymbol{h}_{K} \in \mathbb{C}^{M}$, with spatial covariance matrices $\boldsymbol{R}_{k}=\mathrm{E}\left[\boldsymbol{h}_{k} \boldsymbol{h}_{k}^{H}\right]$. The superimposed signals at the array output are received by a bank of linear filters $\boldsymbol{u}_{1}, \ldots, \boldsymbol{u}_{K}$ (the 'beamformers'). The output of the $k$ th beamformer is

$$
y_{k}=\boldsymbol{u}_{k}^{H}\left(\sum_{1 \leq l \leq K} \boldsymbol{h}_{l} s_{l}+\boldsymbol{n}\right),
$$

where $\boldsymbol{n} \in \mathbb{C}^{M}$ is an AWGN vector, with $\mathrm{E}\left[\boldsymbol{n} \boldsymbol{n}^{H}\right]=\sigma^{2} \boldsymbol{I}$. The coupling coefficients of the $k$ th user are

$$
\left[\boldsymbol{v}_{k}\left(\boldsymbol{u}_{k}\right)\right]_{l}= \begin{cases}\frac{\boldsymbol{u}_{k}^{H} \boldsymbol{R}_{l} \boldsymbol{u}_{k}}{\boldsymbol{u}_{k}^{H} \boldsymbol{R}_{k} \boldsymbol{u}_{k}} & 1 \leq l \leq K, l \neq k \\ \frac{\left\|\boldsymbol{u}_{k}\right\|^{2}}{\boldsymbol{u}_{k}^{H} \boldsymbol{R}_{k} \boldsymbol{u}_{k}} & l=K+1, \\ 0 & l=k .\end{cases}
$$

With the commonly used normalization $\left\|\boldsymbol{u}_{k}\right\|_{2}=1$, the interference function for the beamforming case is

$$
\begin{aligned}
\mathcal{I}_{k}(\underline{\boldsymbol{p}}) & =\left[\max _{\left\|\boldsymbol{u}_{k}\right\|_{2}=1} \frac{\boldsymbol{u}_{k}^{H} \boldsymbol{R}_{k} \boldsymbol{u}_{k}}{\boldsymbol{u}_{k}^{H}\left(\sum_{l \neq k} p_{l} \boldsymbol{R}_{l}+\sigma^{2} \boldsymbol{I}\right) \boldsymbol{u}_{k}}\right]^{-1} \\
& =\min _{\left\|\boldsymbol{u}_{k}\right\|_{2}=1} \underline{\boldsymbol{p}}^{T} \boldsymbol{v}_{k}\left(\boldsymbol{u}_{k}\right) .
\end{aligned}
$$

It can be observed that the interference coupling is not constant. For any power vector $\boldsymbol{p}>0$, the beamformer $\boldsymbol{u}_{k}$ adapts to the interference in such a way that the signalto-interference-plus-noise ratio (SINR) is maximized. This optimization can be solved efficiently via an eigenvalue decomposition. For deterministic channels $\boldsymbol{h}_{1}, \ldots, \boldsymbol{h}_{K}$, we have $\boldsymbol{R}_{l}=\boldsymbol{h}_{l} \boldsymbol{h}_{l}^{H}$, so the interference resulting from optimum beamformers is obtained in closed form

$$
\mathcal{I}_{k}(\underline{\boldsymbol{p}})=\frac{1}{\boldsymbol{h}_{k}^{H}\left(\sigma^{2} \boldsymbol{I}+\sum_{l \neq k} p_{l} \boldsymbol{h}_{l} \boldsymbol{h}_{l}^{H}\right)^{-1} \boldsymbol{h}_{k}} .
$$

For a downlink scenario, we can exploit the reciprocity between uplink and downlink channels. So optimal downlink beamformers can be found indirectly via a "virtual uplink" channel [9].

\section{REFERENCES}

[1] J. M. Aein, "Power balancing in systems employing frequency reuse," COMSAT Tech. Rev., vol. 3, no. 2, pp. 277-300, 1973.

[2] G. Montalbano, I. Ghauri, and D. T. M. Slock, "Spatio-temporal array processing for CDMA/SDMA downlink transmission," in Proc. Asilomar Conf. on Signals, Systems and Computers, Monterey, 1998, pp. 13371341.

[3] M. Schubert and H. Boche, "SIR balancing for multiuser downlink beamforming - a convergence analysis," in Proc. IEEE Int. Conf. on Comm. (ICC). New York, USA, Apr. 2002, pp. 841 - 845 vol.2.

[4] — Q Q - Q Based Resource Allocation and Transceiver Optimization. Foundations and Trends in Communications and Information Theory, 2005/2006, vol. 2, no. 6 .

[5] H. Boche and M. Schubert, "Perron-root minimization for interferencecoupled systems with adaptive receive strategies," IEEE Trans. Commun., vol. 57 , no. 10 , pp. $3173-3164$, Oct. 2009.

[6] - "A calculus for log-convex interference functions," IEEE Trans. Inform. Theory, vol. 54, no. 12, pp. 5469-5490, Dec. 2008.

[7] R. D. Yates, "A framework for uplink power control in cellular radio systems," IEEE J. Select. Areas Commun., vol. 13, no. 7, pp. 1341-1348, Sept. 1995.

[8] H. Boche and M. Schubert, "A unifying approach to interference modeling for wireless networks," IEEE Trans. Signal Processing, vol. 58, no. 6, June 2010.

[9] M. Schubert and H. Boche, "Solution of the multi-user downlink beamforming problem with individual SINR constraints," IEEE Trans. Veh. Technol., vol. 53, no. 1, pp. 18-28, Jan. 2004. 